\title{
BMJ Global Health Completeness and quality of low back pain prevalence data in the Global Burden of Disease Study 2017
}

\author{
Mamata Tamrakar, Priti Kharel, Adrian Traeger, Chris Maher (D) , Mary O'Keeffe, \\ Giovanni Ferreira
}

To cite: Tamrakar M, Kharel $\mathrm{P}$ Traeger A, et al. Completeness and quality of low back pain prevalence data in the Global Burden of Disease Study 2017. BMJ Global Health 2021;6:e005847. doi:10.1136/ bmjgh-2021-005847

Handling editor Seye Abimbola

- Additional online supplemental material is published online only. To view, please visit the journal online (http://dx.doi.org/10.1136/ bmjgh-2021-005847)

Received 28 March 2021 Revised 25 April 2021

Accepted 26 April 2021
Check for updates

(c) Author(s) (or their employer(s)) 2021. Re-use permitted under CC BY-NC. No commercial re-use. See rights and permissions. Published by BMJ

Institute for Musculoskeletal Health, The University of Sydney, Sydney, New South Wales, Australia

Correspondence to Professor Chris Maher; christopher.maher@sydney. edu.au

\section{ABSTRACT}

Introduction Completeness of Global Burden of Disease (GBD) Study data is acknowledged as a limitation. To date, no study has evaluated this issue for low back pain, a leading contributor to disease burden globally.

Methods We retrieved reports, in any language, based on citation details from the GBD 2017 study website. Pairs of raters independently extracted the following data: number of prevalence reports tallied across countries, age groups, gender and years from 1987 to 2017. We also considered if studies enrolled a representative sample and/or used an acceptable measure of low back pain.

Results We retrieved 488 country-level reports that provide prevalence data for 103 of 204 countries (50.5\%), with most prevalence reports $(61 \%)$ being for high-income countries. Only 16 countries $(7.8 \%)$ have prevalence reports for each of the three decades of the GBD. Most of the reports $(79 \%)$ did not use an acceptable measure of low back pain when estimating prevalence.

Conclusion We found incomplete coverage across countries and time, and limitations in the primary prevalence studies included in the GBD 2017 study. This means there is considerable uncertainty about GBD estimates of low back pain prevalence and the disease burden metrics derived from prevalence.

\section{INTRODUCTION}

The Global Burden of Disease (GBD) Study aims to provide disease metrics to understand trends in global health. GBD studies ${ }^{12}$ have become the gold standard for measuring the burden of disease. As an example, the Lancet Low Back Pain Series used GBD disease metrics to argue for the urgent need for action to address the burden and impact of low back pain (LBP) ${ }^{34}$ globally. The challenge is that understanding trends in global health requires an enormous amount of data. As an illustration, the GBD $2019^{2}$ study provides estimates for disease burden expressed in years of life lost, years lived with disability (YLD) and disability-adjusted life years (DALYs) for 369 conditions in 204 countries for the period $1990-2019$. That is $\sim 6.8$ million data points.

\section{Key questions}

What is already known?

- In Global Burden of Disease (GBD) Studies, low back pain (LBP) is usually ranked \#1 when disease burden is measured by disability and among the top 10 when both death and disability are considered.

- A limitation of GBD Studies is the reliance on modelling when data are not available.

What are the new findings?

- The reports included in the GBD 2017 study only provide LBP prevalence data for 103 of 204 countries $(50.5 \%)$ in the time period 1987-2017 and most $(61 \%)$ of the prevalence reports are for highincome countries.

- For the time periods 1987-1996, 1997-2006 and 2007-2017, there were reports for 18, 93 and 55 countries, respectively, with only 16 countries $(7.8 \%)$ having prevalence reports for all three time periods.

- While most reports enrolled a representative sample $(91 \%)$, few (16\%) used an acceptable LBP definition.

What do the new findings imply?

- There is considerable uncertainty about the LBP prevalence estimates in the GBD study because of incomplete coverage and the limitations of the primary prevalence studies.

- This uncertainty will impact two of the three disease burden metrics that are produced by the GBD study: years lived with disability and disability-adjusted life years.

- Uncertainty in GBD estimates means that the true burden of LBP is not known creating a challenge for assessing if progress is being made in reducing the global burden of LBP.

The GBD study uses a complex modelling approach to provide estimates of disease burden. For example, when estimating nonfatal health outcomes the model includes study-level covariates, location-level covariates, disability weights and comorbidity correction, in addition to prevalence data from the primary prevalence studies. The ambitious scope of GBD studies means that 
completeness of data needs to be borne in mind when considering GBD disease metrics. The GBD studies ${ }^{25}$ acknowledge a major limitation: a reliance on modelling to estimate key disease metrics such as incidence and prevalence when primary data are not available. The completeness of prevalence data is particularly important as these data are combined with disability weights to estimate the two burden measures that incorporate disability burden: YLD and DALYs.

While GBD studies acknowledge problems with data completeness, there has been little exploration of the coverage of prevalence data across years, diseases and global regions. The GBD 2017 study $^{5}$ provides a visual portrayal of non-fatal data coverage over time and regions revealing for example that Oceania has less data than Western Europe. What is missing however is a quantitative assessment of data completeness and information on data coverage across years and for individual diseases. This information is important as successive versions of the GBD studies report the leading causes of disease burden for that report year and also trends over time.

Across the various GBD studies, LBP is usually the leading contributor to disease burden expressed as YLD and among the top 10 when disease burden is measured by DALYs. GBD estimates have been used to examine trends in the burden of LBP. For example, $\mathrm{Wu}$ et $\mathrm{al}^{6}$ reported that LBP prevalence reduced slightly in the period between 1990 and 2017, the prevalence was higher in females, increases with age, and that prevalence was higher in Southern Latin America (13.47\%) than in Central Latin America (5.62\%). But even this simple level of analysis requires $>20000$ data points (ie, 21 regions $\mathrm{x} 28$ years $\times 19$ year age bands $\times 2$ genders) and analysis at the level of country raises this to $>200000$ disease metrics. While the authors acknowledge uncertainty in their estimates: 'burden estimates were heavily reliant on models'; they do not quantify the extent of missing primary data. Therefore, it is unclear to what extent the trends identified are driven by primary LBP prevalence data or modelled LBP prevalence data.

The current study examined the completeness of primary LBP prevalence data that informed the estimates in the GBD 2017 study. We also aimed to determine where primary data are lacking and how future prevalence studies contributing data to the GBD studies could be improved.

\section{METHODS}

To describe completeness we tallied the number of reports included in the GBD 2017 study that provided LBP prevalence data for each of the following strata: world regions, countries, years (from 1987 to 2017), age of participants (5-year age groups from birth up to 95+) and gender of participants. We also tallied the subsets of reports that enrolled a representative sample and/or used an 'acceptable' measure of an LBP episode, which we describe below.
Table 1 Characteristics of included primary prevalence study reports $(n=488)$ providing data for the period 19872017

\begin{tabular}{ll}
\hline Variables & N (\%) \\
\hline Study type & \\
Peer-reviewed & $194(40)$ \\
Report or survey & $294(60)$
\end{tabular}

Region (Global Burden of Disease super

regions)

Central Europe, Eastern Europe and Central
Asia

High Income $\quad 300$ (61)

$\begin{array}{lr}\text { Latin America and Caribbean } & 27(6) \\ \text { North Africa and Middle East } & 20(4) \\ \text { South Asia } & 9(2) \\ \text { Southeast Asia, East Asia and Oceania } & 25(5) \\ \text { Sub-Saharan Africa } & 32(7) \\ \text { Prevalence } & \end{array}$

Prevalence

\begin{tabular}{|c|c|}
\hline Any prevalence period & 484 (99) \\
\hline Unclear prevalence period & $4(1)$ \\
\hline Point prevalence & $57(12)$ \\
\hline Incidence & $9(2)$ \\
\hline \multicolumn{2}{|l|}{ Age groups } \\
\hline $0-4$ & $28(6)$ \\
\hline $5-9$ & $33(7)$ \\
\hline $10-14$ & $214(44)$ \\
\hline $15-19$ & $385(79)$ \\
\hline $20-24$ & $256(52)$ \\
\hline $25-29$ & $264(54)$ \\
\hline $30-34$ & $270(55)$ \\
\hline $35-39$ & $273(56)$ \\
\hline $40-44$ & $281(58)$ \\
\hline $45-49$ & $287(59)$ \\
\hline $50-54$ & $297(61)$ \\
\hline $55-59$ & $294(60)$ \\
\hline $60-64$ & $286(59)$ \\
\hline $65-69$ & $276(57)$ \\
\hline $70-74$ & $268(55)$ \\
\hline $75-79$ & $257(53)$ \\
\hline $80-84$ & $245(50)$ \\
\hline $85-89$ & $243(50)$ \\
\hline $90-94$ & $241(49)$ \\
\hline $95+$ & $240(49)$ \\
\hline Data for both genders available & $472(97)$ \\
\hline Representative sample & $446(91)$ \\
\hline Acceptable low back pain definition & $79(16)$ \\
\hline
\end{tabular}

*Numbers do not add up to the total number of reports (488) because some reports measured more than one prevalence period. 


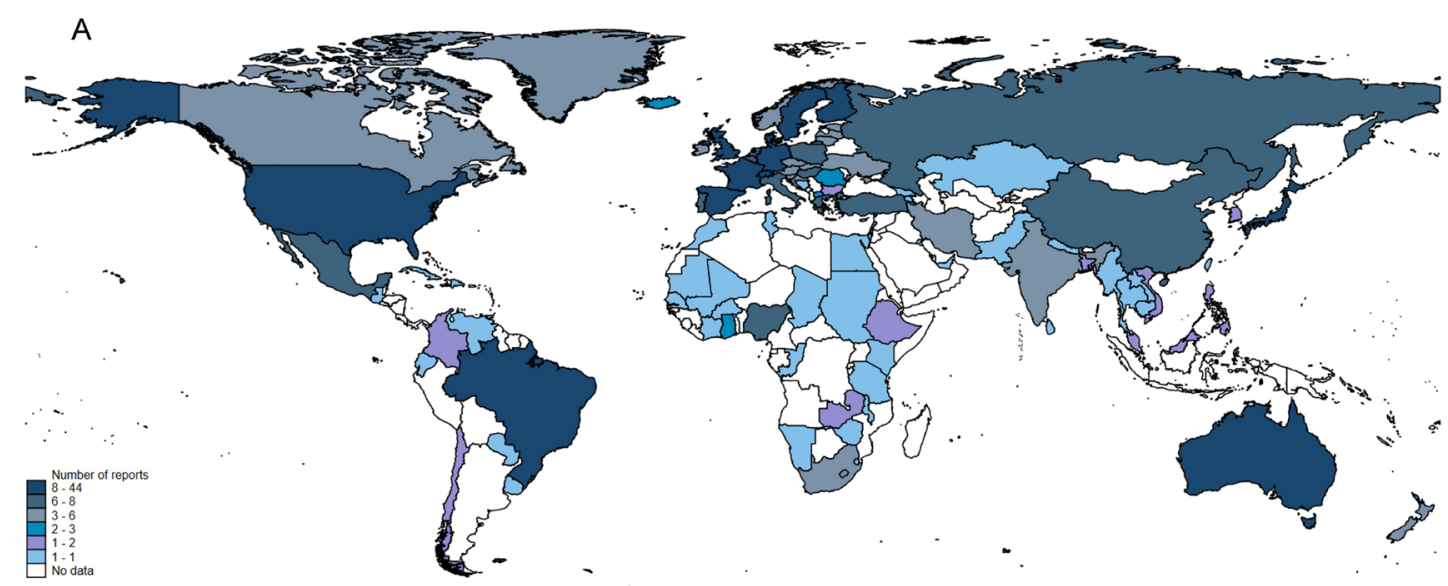

B

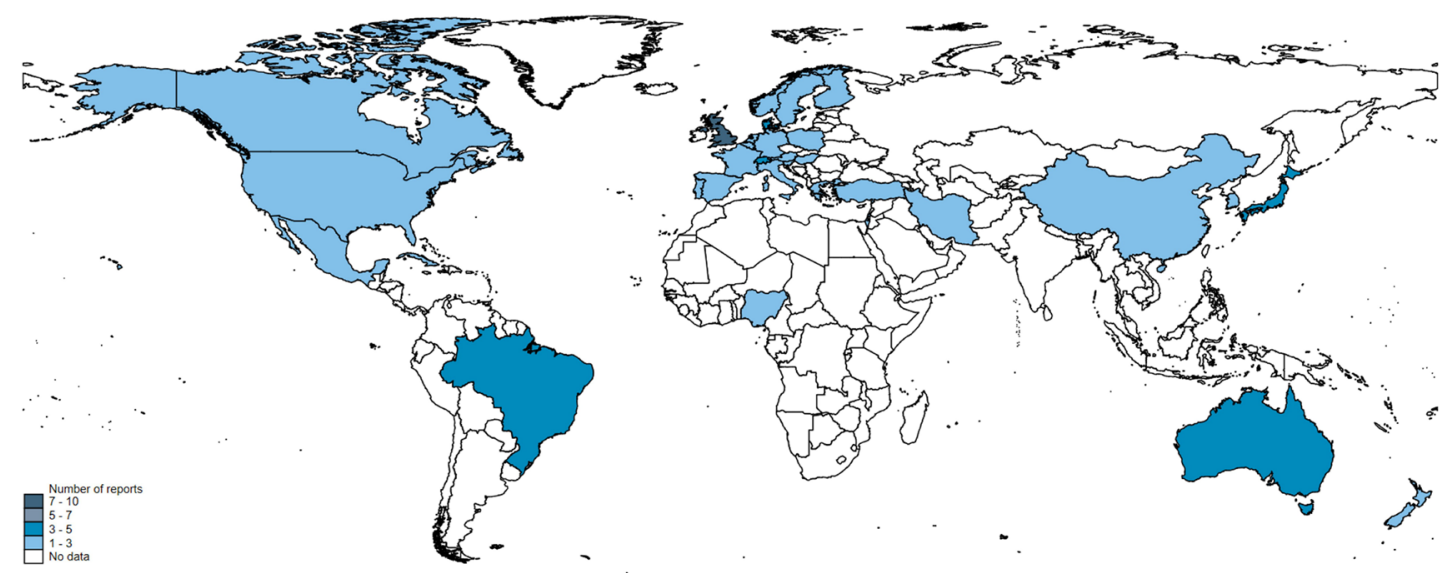

Figure 1 Number of LBP prevalence studies per country (A) and number of LBP prevalence studies per country that used an acceptable measure of LBP (B). LBP, low back pain.

\section{Study design}

This was a descriptive study.

\section{Study procedure}

\section{Search strategy}

We identified citation details for the primary prevalence study reports included in the GBD 2017 study via the Global Health Data Exchange website http://ghdx. healthdata.org/gbd-2017. We searched for data sources using their Data Input Sources Tool. In that tool, we selected 'Nonfatal Health Outcomes' under components AND 'Low Back Pain' under causes. This search revealed all sources used to calculate disease burden metrics used by GBD 2017. These sources included articles published in peer-reviewed journals and unpublished primary reports (eg, government reports, national health surveys, reports of claims data). We searched for the full text of primary reports published in academic journals via electronic databases (eg, PubMed, MEDLINE, EMBASE) and primary reports not published in academic journals via Google and Google Scholar.

\section{Inclusion criteria}

All reports in cluded in the GBD 2017 study were considered eligible for this study.

\section{Data extraction}

Pairs of independent raters, from a panel of four, extracted data from the included reports using a piloted spreadsheet. We included all reports irrespective of language. For languages other than English, we sought help from native speakers in that language who performed the data extraction individually when possible. When we could not find a native speaker, we used the Google translate tool which is considered an accurate tool. ${ }^{7}$ Disagreements were discussed by the two original raters until consensus was reached. If consensus could not be reached a third author arbitrated.

We used a data extraction template to extract the following data from each report: age (5-year age groups from birth up to 95+) and gender of participants, global region/country where the study was conducted, prevalence study (yes/no), prevalence period (point, 1 week, 1 month, 3 months, 6 months, 12 months), incidence study (yes/no), years of prevalence/incidence data contained in each study from 1987 to 2017 , whether the study enrolled a representative sample, and whether the study used an acceptable measure of an LBP episode.

As studies and reports frequently collapsed one or more age groups together (eg, 'age 65+'), we assumed 
Table 2 Countries ( $n=33$ ) with at least one report using an acceptable LBP definition for measuring low back prevalence and frequency of reports providing data for the period 1987-2017

\begin{tabular}{|c|c|}
\hline Region & $\mathbf{N}$ \\
\hline \multicolumn{2}{|c|}{ Central Europe, Eastern Europe and Central Asia } \\
\hline Hungary & 1 \\
\hline Poland & 1 \\
\hline Slovenia & 1 \\
\hline \multicolumn{2}{|l|}{ High Income } \\
\hline Australia & 4 \\
\hline Austria & 1 \\
\hline Belgium & 1 \\
\hline Canada & 1 \\
\hline Denmark & 5 \\
\hline Finland & 3 \\
\hline France & 2 \\
\hline Germany & 2 \\
\hline Greece & 3 \\
\hline Israel & 1 \\
\hline Italy & 1 \\
\hline Japan & 5 \\
\hline Netherlands & 3 \\
\hline New Zealand & 3 \\
\hline Norway & 1 \\
\hline Portugal & 1 \\
\hline South Korea & 1 \\
\hline Spain & 3 \\
\hline Sweden & 2 \\
\hline Switzerland & 5 \\
\hline UK & 10 \\
\hline USA & 2 \\
\hline
\end{tabular}

Latin America and Caribbean

\begin{tabular}{lc} 
Brazil & 5 \\
\hline Cuba & 1 \\
Mexico & 1 \\
North Africa and Middle East & 2 \\
\hline Iran & 1 \\
Turkey & 2 \\
Southeast Asia, East Asia and Oceania & 1 \\
China & \\
Hong Kong & 3 \\
Sub-Saharan Africa & 79 \\
Nigeria & \\
\hline Total & \\
\hline
\end{tabular}

LBP, low back pain.

that data for all age groups had been collected. For each report, we recorded when data collection took place for example, the year 1990. The date for data collection had to be explicitly mentioned in the report otherwise we coded it as unclear. We did not use publication date as a surrogate for this variable.

To be coded 'yes' for an acceptable measure of an LBP episode the survey instrument used in the primary study needed to: (1) include a visual or verbal depiction of the low back region (lower ribs to buttock crease); (2) collect responses directly from the person rather than a proxy and (3) measure an episode of pain OR activity-limiting pain. The operational definitions for enrolling a representative sample are in online supplemental appendix 1.

\section{Data analyses}

We analysed data by country, not report, as some reports provided data for more than one country. We collapsed data by country and tallied the number of prevalence studies across the seven GBD Super Regions. (1) Central Europe, Eastern Europe and Central Asia; (2) High Income; (3) Latin America and Caribbean; (4) North Africa and Middle East; (5) South Asia; (6) Southeast Asia, East Asia and Oceania; (7) sub-Saharan Africa, age groups (in 5-year bands) and over time in 1-year increments from 1987 to 2017. We divided the period covered by GBD 2017 into three time periods 1987-1996, 1997-2006, 2007-2017 (NB the third period is 11 years) to assess the extent to which there were sufficient prevalence reports over time. Our primary analysis was based on the full set of retrieved prevalence reports. We also considered the subset of reports that enrolled a representative sample and/or used an acceptable definition of LBP when measuring prevalence. Choropleth maps were created to summarise the frequency of reports per country. Darker shades represent a higher number of reports. To assess completeness of data per country from 1987 to 2017, we built heatplots to depict the number of studies or reports with data for each of the 30 years that were part of our analysis. White spots mean there is no data available. All analyses were done in Stata V.16.0.

\section{RESULTS \\ Characteristics of identified reports}

We obtained citation details for 470 reports from the data query tool on the GBD website. We located copies of 459 reports (citations for 11 missing reports are in online supplemental appendix 2) and with some reports covering more than one country we finally had 488 country-level reports.

The characteristics of the 488 retrieved countrylevel reports are provided in table 1 . Forty per cent of reports were published in peer-reviewed journals, $12 \%$ reported point prevalence data, the remainder used other or unclear prevalence periods. There were $300(61 \%)$ reports providing data for the GBD Super 
A

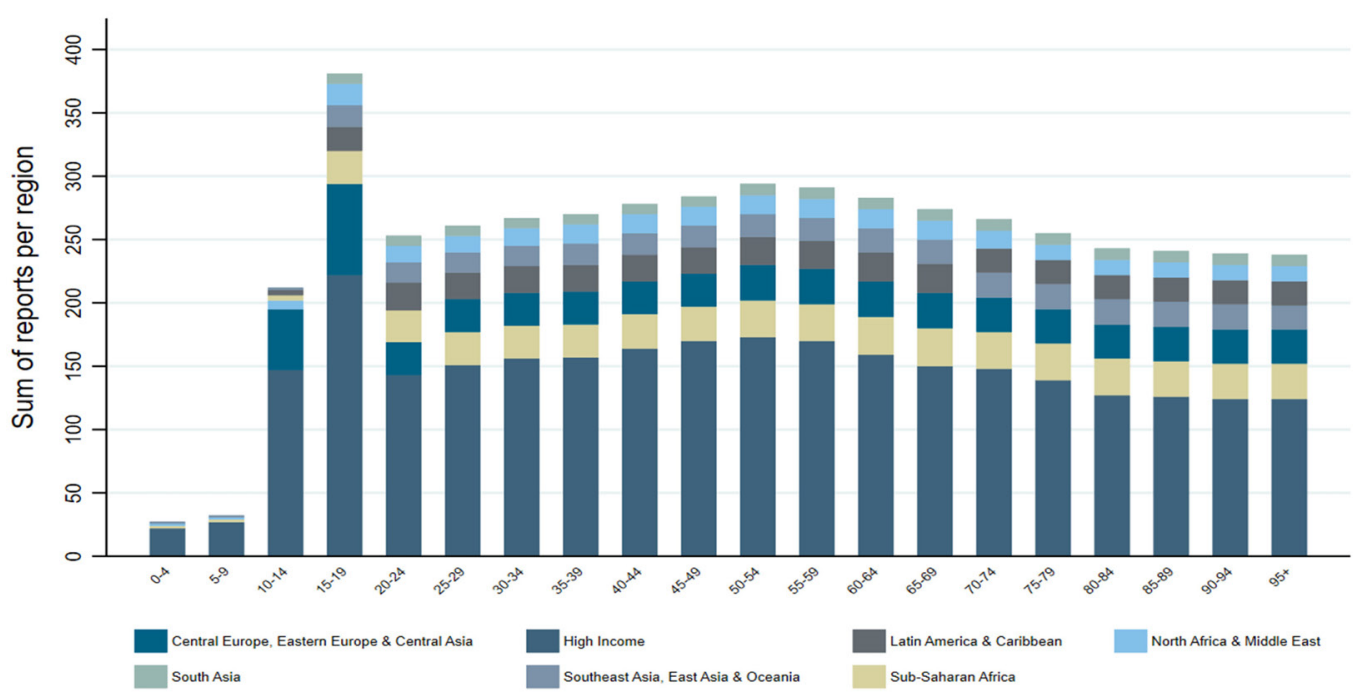

B

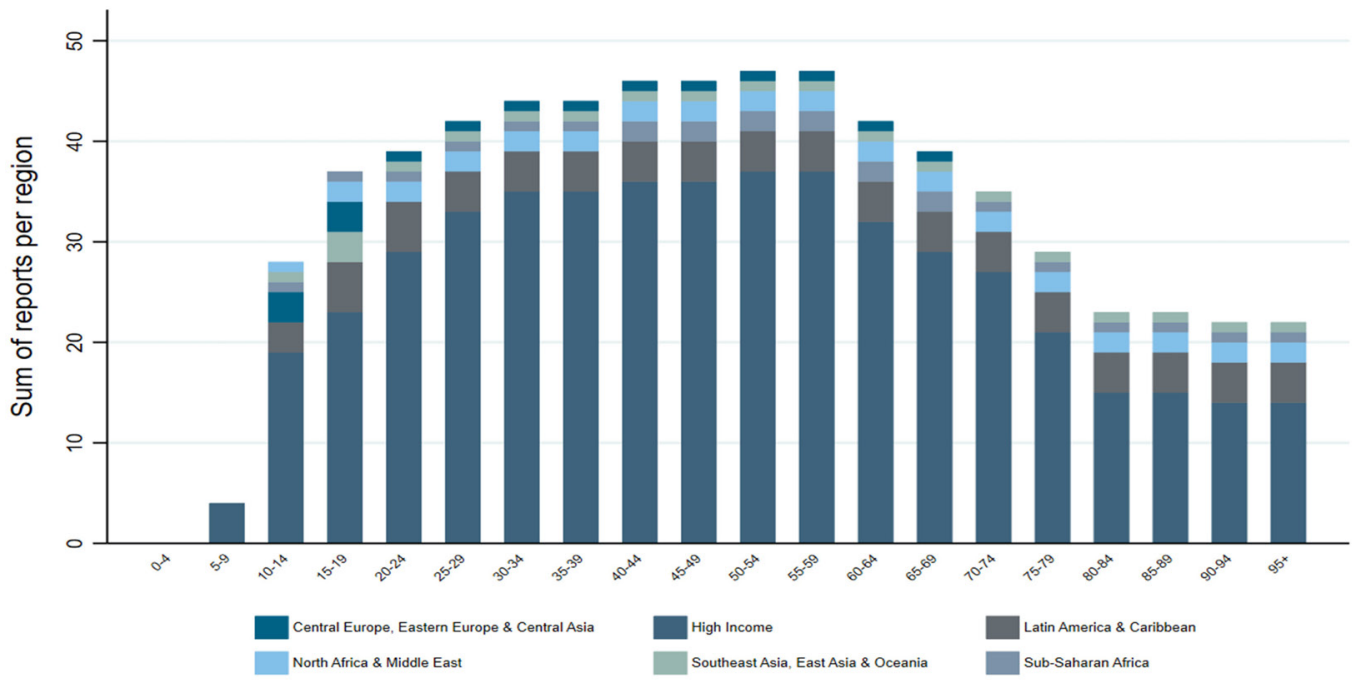

Figure 2 Number of LBP prevalence studies by age group per region (A) and number of LBP prevalence studies by age group per region that used an acceptable measure of LBP (B). LBP, low back pain.

Region 'high-income' countries, the other six global regions had from 9 to 75 reports providing prevalence data. While most studies $(97 \%)$ provided data for males and females that was not the case across age groups with coverage ranging from 28 studies for age group 0-4 years to 385 studies for age group 15-19 years.

At the country level, 103 of 204 countries (50.5\%) had at least one prevalence report (figure 1A). There was at least one prevalence report for 18 of 204 countries $(8.8 \%)$ in the period 1987-1996, 93 of 204 (45.6\%) countries in the second period 1997-2006 and 55 of 204 countries $(26.9 \%)$ in the third period 2007-2017. Only
16 of $204(7.8 \%)$ countries had a prevalence report in each time period.

\section{Coverage based on prevalence reports that used an acceptable definition of LBP}

At the region level, all seven had at least one primary prevalence report but only 33 of 204 countries (16.2\%) had a primary prevalence report (table 2). High income was the region with the best coverage (60 reports) but within that region countries had from 1 to 10 reports. For the other six regions there was very little prevalence data. Country-level coverage is portrayed in figure 1B. For example, there are only two primary reports for China, 


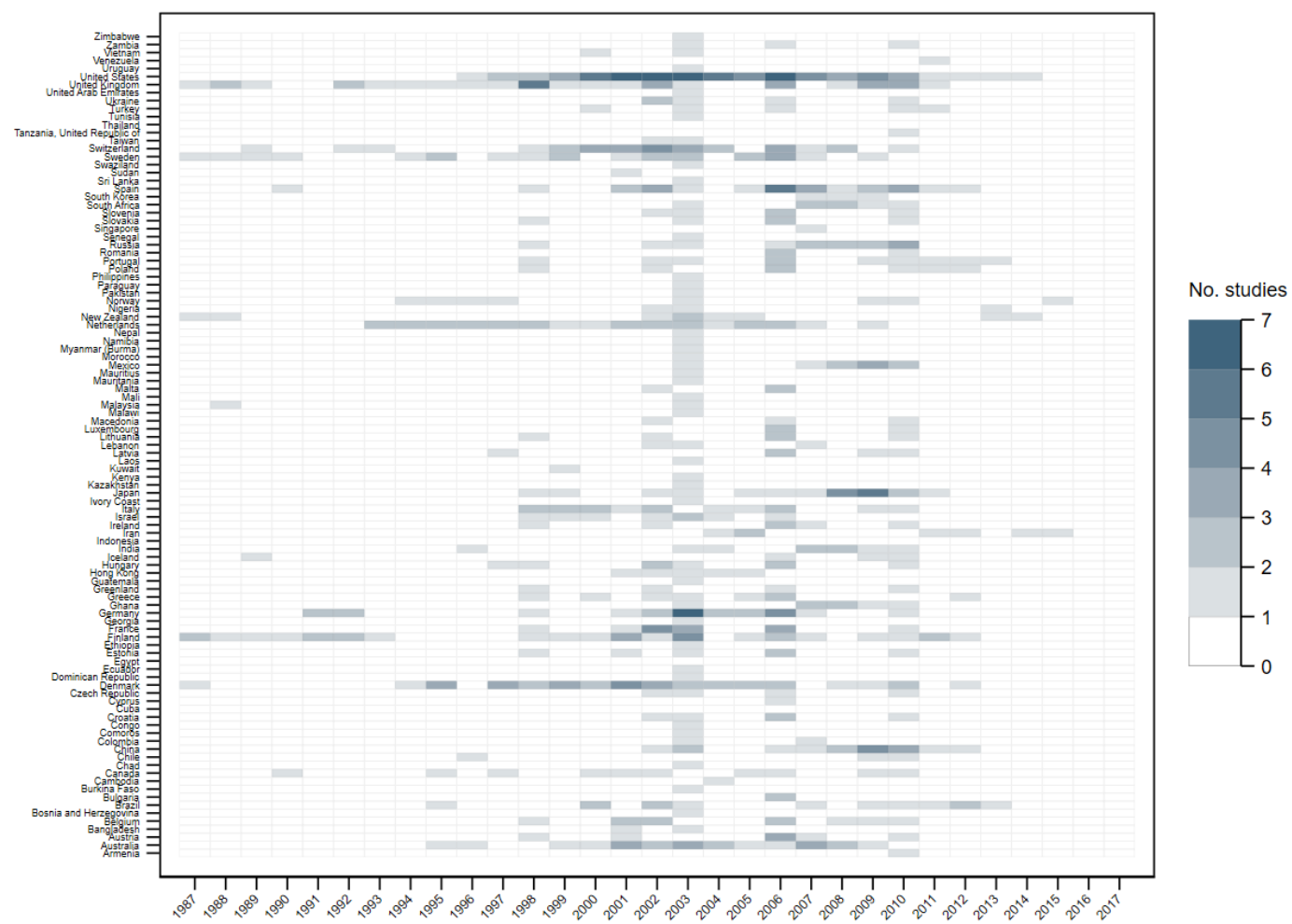

Figure 3 Number of LBP prevalence studies per year across countries from 1987 to 2017. LBP, low back pain.

and no primary reports for India and Brazil is the only South American country with data based on an acceptable LBP definition.

\section{Coverage across age groups}

The number of reports per 5-year age groups, stratified by global region is shown in figure $2 \mathrm{~A}$ (all reports) and figure 2B (reports using an acceptable definition of LBP). The prevalence data from those studies using an acceptable definition of LBP is skewed towards younger adults and a greater proportion of the data comes from high-income countries.

\section{Coverage over time}

The completeness of data per country from 1987 to 2017 is shown in figure 3. Limited data mean it is challenging to provide comments about the prevalence of LBP over time at the level of a country. For example the USA has data for the years 1999, 2000 and 2006 in the period 1987-2017, but Canada only has data for the year 1995 .

\section{DISCUSSION}

\section{Statement of principal findings}

The prevalence reports included in the GBD 2017 study only provide prevalence data for 103 of 204 countries $(50.5 \%)$ in the time period 1987-2017. For the time periods 1987-1996, 1997-2006 and 2007-2017; there were reports for 18, 93 and 55 countries respectively. Only 16 countries $(7.8 \%)$ have prevalence reports for all three time periods. Most of the prevalence reports are for high-income countries. While most reports enrolled a representative sample, few used an acceptable LBP definition.

\section{Strengths and weaknesses of the study}

The strengths of the study include that we piloted the data extraction template until $100 \%$ agreement between raters was achieved. We also used double data extraction to ensure data quality. A weakness of the study is that while we performed a comprehensive search for the reports included in the GBD 2017 study and retrieved $98 \%$; there were 11 reports that we could not locate. Another potential weakness is that the GBD 2019 study has been released; however we do not think our main result about coverage would change as the 2019 study has 455 reports compared with 470 reports in the 2017 study. This small decrease in studies would not be enough to change our main results. When study reports presented results stratified by age and used wider categories than we did for example, age $65+$, we presumed data for all age bands had been collected. This approach will exaggerate coverage across age bands.

\section{Strengths and weaknesses in relation to other studies, discussing important differences in results}

The trends in LBP prevalence reported in previous analyses of GBD estimates need careful consideration. For example $\mathrm{Wu}$ et a ${ }^{6}$ analysed the GBD 2017 study estimates and concluded that for the year 2017 there were marked differences in point prevalence across global regions 
ranging from $13.47 \%$ in Southern Latin America to 3.92\% in East Asia. However, we found that these two regions had little if any acceptable primary prevalence data in the period 1987-2017, and no primary prevalence data of any sort for the year 2017. Another issue that complicates drawing conclusions about point prevalence is that only $12 \%$ of the LBP prevalence studies included in the GBD 2017 study directly measured point prevalence. A challenge for future research in this area is to communicate to readers the extent to which the presented point prevalence estimates incorporate indirect data (ie, other prevalence periods, bodily pain rather than back pain) and/or modelled data. At present there is little transparency on these two important issues.

\section{Meaning of the study: possible explanations and implications for clinicians and policymakers}

The limited prevalence data we have means that it is challenging to assess if progress is being made in reducing the global burden of LBP. Only 16 countries have prevalence data for each decade of the GBD 2017 study, and for almost half the countries there are no data at all. Given the enormous amount of money that is currently devoted to healthcare for LBP there would seem to be a strong justification for better surveillance to understand if these investments are achieving their intended effects.

While we studied completeness of LBP prevalence data, similar issues likely apply to other common health conditions. When we repeated our search strategy for the 25 most burdensome health conditions in the GBD 2019 study (judged by DALYs); we found that most conditions had less prevalence reports than were available for LBP. The number of reports ranged from 19 (Dietary iron deficiency) to 2644 (Malaria) with a median of 429 (see online supplemental appendix 3). This situation makes the GBD study tables of leading causes of disease burden challenging to interpret.

The GBD model used to estimate non-fatal health outcomes is not only reliant on the completeness and validity of prevalence data. There is one set of disability weights, representing different severity levels of LBP, with no attempt to generate disability weights per country. This approach presumes that cases of LBP are similarly disabling regardless of where the person lives, whereas the prevailing view in the LBP field is that disability can vary substantially between countries and is influenced by factors such as work, health and social systems. ${ }^{3}$ In this regard, it is important to note that the LBP disability weights used in the GBD studies are based on surveys of respondents mainly from high-income settings. ${ }^{8}$ A second issue is that the primary prevalence reports measure prevalence of LBP, not prevalence of the six different types of LBP represented by the set of six disability weights used in the model. The split across the severity types is estimated from US claims data; which may not generalise to people who do not seek care (approximately $42 \%$ of people with LBP $)^{9}$ or other countries.

\section{Unanswered questions and future research}

Our results strongly suggest we need better primary studies that adopt agreed best practice methods for measuring LBP prevalence that are implemented at regular time intervals. There is also a need to focus energies on parts of the world that have little or no LBP prevalence data. ${ }^{10}$ A good example of a model is that adopted in the Solomon Islands ${ }^{11}$ where the Global Alliance for Musculoskeletal Health Survey Module was included in the country's national health survey. Another option would be to include the Musculoskeletal Health Survey Module in the WHO's STEPs programme for non-communicable diseases. $^{12}$

\section{CONCLUSION}

Our results suggest there is considerable uncertainty about the LBP prevalence estimates in the 2017 GBD study because of incomplete coverage and the limitations of the primary prevalence studies. The limited prevalence data are concerning as prevalence data drive two of the three disease burden metrics produced by the GBD study: YLD and DALYs.

Twitter Chris Maher @CGMMaher and Giovanni Ferreira @giovanni_ef

Contributors All authors contributed equally.

Funding The authors have not declared a specific grant for this research from any funding agency in the public, commercial or not-for-profit sectors.

Map disclaimer The depiction of boundaries on the map(s) in this article does not imply the expression of any opinion whatsoever on the part of BMJ (or any member of its group) concerning the legal status of any country, territory, jurisdiction or area or of its authorities. The map(s) are provided without any warranty of any kind, either express or implied.

Competing interests AT reports he has received research grants from numerous Government and not for profit agencies outside the submitted work. CM reports he has received research grants from numerous Government and not for profit agencies outside the submitted work. His expenses have been covered by professional associations hosting conferences he has spoken at. Flexeze provide heat wraps at no cost for the SHaPED trial that he is an investigator on. M0 received a Marie Curie Fellowship from the European Commission from 2018 to 2020, outside the submitted work. MT has nothing to disclose. Kharel has nothing to disclose. GF has nothing to disclose

\section{Patient consent for publication Not required.}

Provenance and peer review Not commissioned; externally peer reviewed.

Data availability statement Data are available on request.

Supplemental material This content has been supplied by the author(s). It has not been vetted by BMJ Publishing Group Limited (BMJ) and may not have been peer-reviewed. Any opinions or recommendations discussed are solely those of the author(s) and are not endorsed by BMJ. BMJ disclaims all liability and responsibility arising from any reliance placed on the content. Where the content includes any translated material, BMJ does not warrant the accuracy and reliability of the translations (including but not limited to local regulations, clinical guidelines, terminology, drug names and drug dosages), and is not responsible for any error and/or omissions arising from translation and adaptation or otherwise.

Open access This is an open access article distributed in accordance with the Creative Commons Attribution Non Commercial (CC BY-NC 4.0) license, which permits others to distribute, remix, adapt, build upon this work non-commercially, and license their derivative works on different terms, provided the original work is properly cited, appropriate credit is given, any changes made indicated, and the use is non-commercial. See: http://creativecommons.org/licenses/by-nc/4.0/.

\section{ORCID iD}

Chris Maher http://orcid.org/0000-0002-1628-7857 


\section{REFERENCES}

1 GBD 2019 Viewpoint Collaborators. Five insights from the global burden of disease study 2019. Lancet 2020;396:1135-59.

2 GBD 2019 Diseases and Injuries Collaborators. Global burden of 369 diseases and injuries in 204 countries and territories, 1990-2019: a systematic analysis for the global burden of disease study 2019 . Lancet 2020;396:1204-22.

3 Hartvigsen J, Hancock MJ, Kongsted A, et al. What low back pain is and why we need to pay attention. Lancet 2018;391:2356-67.

4 Buchbinder R, van Tulder M, Öberg B, et al. Low back pain: a call for action. Lancet 2018;391:2384-8.

5 GBD 2017 DALYs and HALE Collaborators. Global, regional, and national disability-adjusted life-years (DALYs) for 359 diseases and injuries and healthy life expectancy (HALE) for 195 countries and territories, 1990-2017: a systematic analysis for the global burden of disease study 2017. Lancet 2018;392:1859-922.

6 Wu A, March L, Zheng X, et al. Global low back pain prevalence and years lived with disability from 1990 to 2017 : estimates from the global burden of disease study 2017. Ann Trans/ Med 2020;8:299.
7 Aiken M. An updated evaluation of Google translate accuracy. Stud Ling Literat 2019;3:p253.

8 Salomon JA, Haagsma JA, Davis A, et al. Disability weights for the global burden of disease 2013 study. Lancet Glob Health 2015;3:e712-23.

9 Ferreira ML, Machado G, Latimer J, et al. Factors defining careseeking in low back pain - a meta-analysis of population based surveys. Eur J Pain 2010;14:747.e1-e7.

10 Ferreira GE, Buchbinder R, Zadro JR, et al. Are musculoskeletal conditions neglected in national health surveys? Rheumatology 2021:keab025.

11 Hoy DG, Raikoti T, Smith E, et al. Use of the global alliance for musculoskeletal health survey module for estimating the population prevalence of musculoskeletal pain: findings from the Solomon Islands. BMC Musculoskelet Disord 2018;19:292.

12 World Health Organization. The steps instrument and support materials, 2021. Available: https://www.who.int/ncds/surveillance/ steps/instrument/en/ 